\title{
Differential expression of CXCR4 and CXCR7 with various stem cell markers in paired human primary and recurrent glioblastomas
}

\author{
CHARLOTTE FLÜH ${ }^{1 *}$, KIRSTEN HATTERMANN ${ }^{2 *}$, H. MAXIMILIAN MEHDORN $^{1}$, \\ MICHAEL SYNOWITZ $^{1}$ and JANKA HELD-FEINDT ${ }^{1}$ \\ ${ }^{1}$ Department of Neurosurgery, University Medical Center Schleswig-Holstein UKSH, Campus Kiel, \\ D-24105 Kiel; ${ }^{2}$ Department of Anatomy, University of Kiel, D-24118 Kiel, Germany
}

Received November 18,2015; Accepted December 27, 2015

DOI: 10.3892/ijo.2016.3354

\begin{abstract}
The chemokine CXCL12 (also termed SDF-1, stromal cell-derived factor-1) and its receptors CXCR4 and CXCR7 are known to play a pivotal role in tumor progression including glioblastomas (GBM). Previous investigations focused on the expression and functional roles of CXCR4 and CXCR7 in different GBM cell subpopulations, but comparative analysis in matched primary versus recurrent GBM samples are still lacking. Thus, here we investigated the expression of CXCR4 and CXCR7 on mRNA and protein level using matched primary and recurrent GBM pairs. Additionally, as GBM CXCR4-positive stem-like cells are supposed to give rise to recurrence, we compared the expression of both receptors in primary and recurrent GBM cells expressing either neural (MUSASHI-1) or embryonic stem cell markers (KLF-4, OCT-4, SOX-2, NANOG). We were able to show that both CXCR4 and CXCR7 were expressed at considerable mRNA and protein levels. CXCR7 was downregulated in relapse cases, and different groups regarding CXCR4/CXCR7 expression differences between primary and recurrent samples could be distinguished. A co-expression of both receptors was rare. In line with this, CXCR4 was co-expressed with all investi-
\end{abstract}

Correspondence to: Dr Janka Held-Feindt, Department of Neurosurgery, University of Schleswig-Holstein Medical Center, Campus Kiel, Arnold-Heller-Str. 3, Building 41, D-24105 Kiel, Germany E-mail: janka.held-feindt@uksh.de

Abbreviations: $\mathrm{C}_{\mathrm{T}}$, cycle of threshold; DAPI, 4',6-diamidino2-phenylindole; DMEM, Dulbecco's modified Eagle's medium; FCS, fetal calf serum; GAPDH, glycerinaldehyde-3-phosphatedehydrogenase; GBM, glioblastoma multiforme; KLF-4, Krüppel-like factor 4; MUSASHI-1, Musashi (Drosophila) homolog 1; NANOG, 'Tir nan Og'; OCT-4, octamer binding transcription factor; qRT-PCR, quantitative reverse transcription polymerase chain reaction; SOX-2, sex determining region Y-box 2; WHO, World Health Organization

Key words: glioblastomas, primary, recurrent, stem cells, CXCR4, CXCR7 gated neural and embryonic stem cell markers in both primary and recurrent tissues, whereas CXCR7 was mostly found on stem cell marker-negative cells, but was co-expressed with KLF-4 on a distinct GBM cell subpopulation. These results point to an individual role of CXCR4 and CXCR7 in stem cell marker-positive GBM cells in glioma progression and underline the opportunity to develop new therapeutic tools for GBM intervention.

\section{Introduction}

Gliomas are the most common type of primary brain tumors. The most malignant form, glioblastoma multiforme (GBM), accounts for $\sim 15 \%$ of all brain tumors, and $>50 \%$ of all astrocytomas (1). Current standard treatment of GBM is surgical resection of the tumor, followed by adjuvant radio- and chemotherapy. However, median survival time for GBM patients is still poor, $12-15$ months despite multimodal therapy (2), and currently there are no effective long-term treatments for this malignancy. One of the primary reasons for the poor prognosis is the development of recurrence composed of highly proliferative and infiltrative tumor cells which massively invade into the surrounding brain parenchyma and contribute to the fatal progression (2).

Chemokines, small chemotactic cytokines, are known to contribute to a broad spectrum of physiological and pathological processes, including angiogenesis (3), haematopoiesis (4), development $(5,6)$ and also tumor initiation, survival and progression $(7,8)$.

In particular, the chemokine CXCL12 (also termed SDF-1, stromal cell-derived factor-1) and its receptors CXCR4 and CXCR7 seem to play a pivotal role in tumor progression, as described for different tumor types including GBM (9-11). In GBM, CXCL12 and CXCR4 are overexpressed in tumor tissues when compared to normal brain parenchyma and their expression level correlates with tumor grade and poor prognosis (12). The CXCL12/CXCR4 activation in glioma cells and specific cells of the surrounding microenvironment (e.g., microglia, endothelial cells, and mesenchymal cells) contributes to GBM proliferation, spreading, and chemoresistance as reviewed for example by Würth et al 
(13). The long known receptor CXCR4 is expressed on glioma cells with stem cell properties $(14,15)$. These cells are able to perform self-renewal, to recapitulate the whole tumor and to differentiate into specific GBM subpopulations. Thus, they are likely responsible for the development of glioblastoma relapses and the poor prognosis of recurrent GBM (16). Nevertheless, not only CXCR4, but also CXCR7, which has been described in tumors, is a regulator of GBM growth (13). For example, CXCR7 is highly expressed in tumor endothelial, microglial and GBM cells $(15,17)$, controls tumor diffusion through CXCL12 gradients and is frequently detected in GBM-associated vasculature (18). Interestingly, in contrast to CXCR4, CXCR7 was detected on more differentiated GBM cells (15). However, a significant correlation between CXCR4 and CXCR7 in GBM was observed (19). These results are fascinating and point to a pivotal role of CXCR4 and CXCR7 in glioma progression including especially the development of recurrences.

\section{Materials and methods}

Tumor specimens. GBM samples were surgically dissected tissues from the Department of Neurosurgery (Kiel, Germany) and were obtained in accordance with the Helsinki Declaration of 1975 with approval of the ethics committee of the University of Kiel, Germany after written informed consent of donors (file reference: D 536/15). Tumors were classified according to the WHO criteria, and the diagnosis was established by a pathologist. A total of 28 GBM (14 primary and 14 recurrent tumors, paired samples for each single donor) was included. If enough material was available, matched probes of individual tumor samples were used for different investigations.

Reverse transcription and real-time PCR ( $R$ RT-PCR). RNA was isolated with the TRIzol reagent (Invitrogen, Carlsbad, CA, USA), digested by DNase, cDNA was synthesized, and quantitative reverse transcription real-time PCR (qRT-PCR) was performed as described before (20) using TaqMan primer probes (Applied Biosystems, Foster City, CA, USA): glycerinaldehyde-3-phosphate-dehydrogenase (hGAPDH) (Hs99999905_m1), hCXCR4 (Hs00237052_m1), hCXCR7 (Hs00664172_s1). Fluorescent data were converted into cycle threshold $\left(\mathrm{C}_{\mathrm{T}}\right)$ measurements, and $\Delta \mathrm{C}_{\mathrm{T}}$ values of each sample were calculated as $\mathrm{CT}_{\text {gene of interest }}-\mathrm{CT}_{\mathrm{GAPDH}}$. Relative gene expression was calculated with $2^{\text {(normalized CT non-stimulated - normalized }}$ CT stimulated) $=n$-fold of control. $\mathrm{A} \Delta \mathrm{C}_{\mathrm{T}}$ value of 3.33 corresponds to one magnitude lower gene expression compared to GAPDH. To visualize possible similarities in chemokine receptor expression of individual primary-recurrent GBM pairs, relative gene expression data were assigned to grey shades in Fig. 1. A relative gene expression value of 1 (= equal expression in primary and recurrent GBM) was assigned as $30 \%$ grey, lower n-fold expression values (lower expression in recurrent compared to primary) were displayed with increasing lighter shading with 0 corresponding to white. Relative expression values $>1$ (higher expression in recurrence compared to primary) were assigned with increasing darker grey shades until 3-fold induction (or higher) which was assigned as maximum (black). Afterwards, a 'heatmap-like' arrangement of individual primary-recurrent GBM pairs was performed orientating to up- or downregulation of CXCR4 and CXCR7 in recurrent samples.
Immunofluorescence. Cryostat sections of different primary and recurrent GBM tissues were fixed in acetone/methanol, and sequentially blocked with Sudan black and $0.1 \%$ bovine serum albumin as described before (20). Primary antibodies were applied overnight at $4^{\circ} \mathrm{C}$, secondary antibodies were incubated at $37^{\circ} \mathrm{C}$ for $1 \mathrm{~h}$, nuclei were counterstained with 4',6-diamidino2-phenylindole (DAPI; Sigma-Aldrich, Hamburg, Germany), slides were embedded with Immumount (ThermoShandon, Pittsburgh, PA, USA) and digital photography was performed using a Zeiss microscope and Zeiss camera (Zeiss, Oberkochen, Germany). Primary antibodies were anti-OCT-4 (octamer binding transcription factor 4; 1:150, rabbit; Cell Signaling, Danvers, MA, USA), anti-SOX-2 (sex determining region Y-box 2; 1:200, rabbit; Santa Cruz Biotechnology, CA, USA), anti-MUSASHI-1 [Musashi (Drosophila) homolog 1; 1:100, mouse; R\&D Systems, Wiesbaden, Germany], anti-NANOG ('Tir nan Og'; 1:500, rabbit; Thermo Fisher Scientific, Rockford, IL, USA), anti-KLF-4 (Krüppel-like factor 4; 1:250, mouse; Thermo Fisher Scientific), anti-CXCR4 (1:200, rabbit; Imgenex IMG 125-2, San Diego, CA, USA) and anti-CXCR7 (1:100, mouse, MAB42273; R\&D Systems). If primary antibodies were derived from the same species, unspecific binding was blocked by $\mathrm{F}(\mathrm{ab})$ fragments derived from this species [donkey anti-mouse and anti-rabbit $\mathrm{F}(\mathrm{ab})$ fragments, 1:100, from Dianova, Hamburg, Germany]. Primary antibodies were omitted for negative controls. As secondary antibodies donkey anti-mouse or anti-rabbit IgGs labeled with Alexa Fluor 488 or Alexa Fluor 555 (1:1,000; Invitrogen) were used.

Statistical analysis. For statistical analyses a two-tailed Student's t-test with matched samples was used. Significance level was $\mathrm{p}<0.05$ (indicated by an asterisk (*) in the figures).

\section{Results}

Expression of CXCR4 and CXCR7 in human primary and recurrent GBM pairs. To evaluate mRNA expression levels of CXCR4 and CXCR7, qRT-PCR analysis was performed using matched probes of solid human primary and recurrent GBM. Results are shown in Fig. 1, where single $\Delta \mathrm{C}_{\mathrm{T}}$ values for primary GBM samples are demonstrated in black circles and $\Delta \mathrm{C}_{\mathrm{T}}$ values for recurrent ones in white triangles. It should be kept in mind that a $\Delta \mathrm{C}_{\mathrm{T}}$ value of 3.33 corresponds to one magnitude lower gene expression.

Irrespective of the tumor identity (primary versus recurrent), both CXCR4 and CXCR7 were detected at considerable levels in solid GBM tissues with CXCR7 clearly to higher extent. Additionally, when comparing primary and recurrent GBM samples, the chemokine receptor CXCR7 was expressed in lower amounts in recurrences (mean reduction to $\sim 40 \%$; $\mathrm{p}<0.05)$, while this was not observed for CXCR4. In detail, the normalized averaged $\Delta \mathrm{C}_{\mathrm{T}}$ values for investigated primary/ recurrent GBM samples were: 7.74/7.91 (CXCR4), and 2.61/3.95 (CXCR7), respectively (Fig. 1, left).

To compare chemokine receptor expression differences between individual primary and recurrent GBM pairs more in detail, we arranged the n-fold expression changes of all individual primary-recurrent GBM pairs as a heatmap: equal n-fold expression in primary and recurrent GBM was assigned as $30 \%$ grey, lower n-fold expression values were displayed 


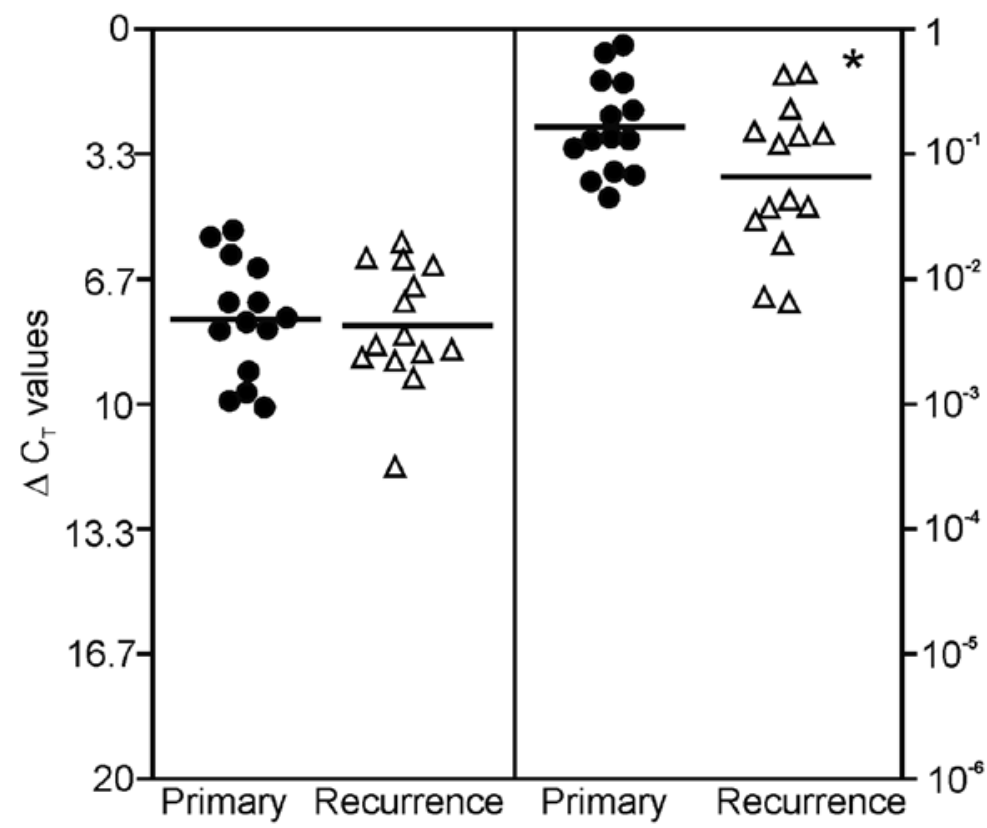

\begin{tabular}{|c|c|}
\hline CXCR4 & CXCR7 \\
\hline 0.01 & 0.19 \\
\hline 0.10 & 0.12 \\
\hline 0.22 & 0.83 \\
\hline 0.42 & 0.28 \\
\hline 0.42 & 0.28 \\
\hline 0.57 & 0.01 \\
\hline 0.89 & 0.59 \\
\hline 1.63 & 0.49 \\
\hline 2.52 & 0.06 \\
\hline 3.03 & 0.64 \\
\hline 7.13 & 1.17 \\
\hline 3.02 & 1.16 \\
\hline 2.22 & 1.77 \\
\hline 12.24 & 6.02 \\
\hline
\end{tabular}

Figure 1. Left, mRNA expression of CXCR4 and CXCR7 in 14 paired primary (black circles) and recurrent (white triangles) glioblastoma samples was evaluated by real-time RT-PCR (logarithmic scale, $\Delta \mathrm{C}_{\mathrm{T}}=3.33$ corresponds to a 10 -fold difference). The receptors CXCR 4 and CXCR7 were detectable in primary and recurrent glioblastoma samples on comparably high levels. In contrast to CXCR4, mean CXCR7 mRNA expression was significantly reduced to $~ 40 \%$ in recurrent glioblastomas ( $" \mathrm{p}<0.05$ ). Right, heatmap-like analysis of $\mathrm{n}$-fold expression differences of CXCR4 and CXCR7 (obtained by qRT-PCR; calculated with $2^{\text {(normalized } C T \text { non-stimulated - normalized CT stimulated) }}=\mathrm{n}$-fold of control) between 14 primary-recurrent glioblastoma pairs. Equal $\mathrm{n}$-fold expression in primary and recurrent pairs was assigned as $30 \%$ grey, lower $\mathrm{n}$-fold expression values with increasing lighter shading with 0 corresponding to white, and relative $\mathrm{n}$-fold expression values $>1$ with increasing darker grey shades until 3-fold induction (or higher) which was assigned as maximum (black). In total three different glioblastoma groups could be distinguished - one with lower mRNA expression of both CXCR4 and CXCR7, one with higher expression amounts of CXCR4 in combination with lower amounts of CXCR7, and one with higher expression amounts of both chemokine receptors in recurrences, respectively.

with increasing lighter shading with 0 corresponding to white, and relative $n$-fold expression values $>1$ were assigned with increasing darker grey shades until 3-fold induction (or higher) which was assigned as maximum (black). By this, three different GBM groups in our collective could be identified (Fig. 1, right): the first group, containing seven primary-recurrent GBM pairs, was characterized by a general lower mRNA expression of CXCR4 and CXCR7 in recurrent samples. The second group, containing five primary-recurrent GBM pairs, was characterized by higher expression of CXCR4 in combination with lower expression of CXCR7. The third group included two GBM pairs and was characterized by higher expression of both chemokine receptors in recurrences. Interestingly, a combination of increased CXCR7 and decreased CXCR4 expression was not observed in our cohort. Summarized, various combinations of loss or gain of CXCR4 and CXCR7 mRNA expression are apparently detectable in our collective of paired primary and recurrent GBM samples, and it became clear that a more precise evaluation of chemokine receptor expression during the progression from primary to recurrent GBM is possible if each individual pair is analyzed in detail.

To confirm qRT-PCR results and to evaluate CXCR4 and CXCR7 expression also on the protein level in primary-recurrent GBM pairs, we performed double-immunofluorencence staining of both chemokine receptors. Nuclei were stained with DAPI. Exemplary results of stained matched primary and recurrent samples are shown in Fig. 2. Although no clear quantitative data could be obtained using fluorescence immunostaining of cryo-sections the primary tumors showed high amounts of CXCR4 or CXCR7 positively stained cells, whereas in matched recurrent samples only few CXCR7positive cells, but considerable amounts of CXCR4-positive cells were visible. In addition, the majority of tumor cells were solely positive for CXCR4 or CXCR7, respectively (exemplified in Fig. 2), only single cells expressed CXCR4 and CXCR7 in the same cell regions [examples for merged regions (yellow) are shown in Fig. 2].

Summarized, while both CXCR4 and CXCR7 are expressed at considerable levels in primary and recurrent GBM samples, CXCR7 expression is apparently downregulated in relapsed cases. Regarding CXCR4/CXCR7 expression differences between primary and recurrent samples, our cohort included different GBM groups of combined induction or reduction upon relapse. Expression of CXCR4 and CXCR7 was confirmed on protein level, and co-stainings revealed single as well as double-positive cells.

Cellular allocation of CXCR4 and CXCR7 in human primary and recurrent GBM pairs. We and others have shown that CXCR4 is predominately expressed in (tumor) stem-like cells $(14,15)$, and GBM stem-like cells are supposed to give rise to the recurrences. Therefore, we analyzed in a next step in more detail which cells might account for expression of CXCR4 and CXCR7 in primary and recurrent GBM. Thus, co-stainings with neural stem/progenitor (RNA-binding protein MUSASHI-1) and different embryonic stem cell markers [KLF-4, OCT-4, SOX-2 as embryonic stem cell transcription 

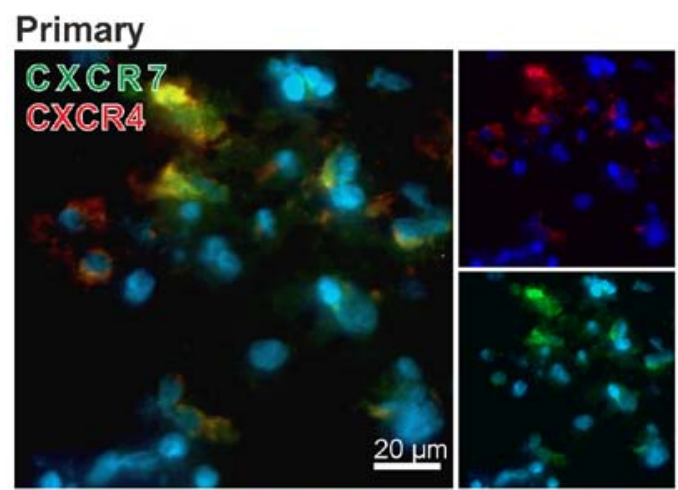

Recurrence
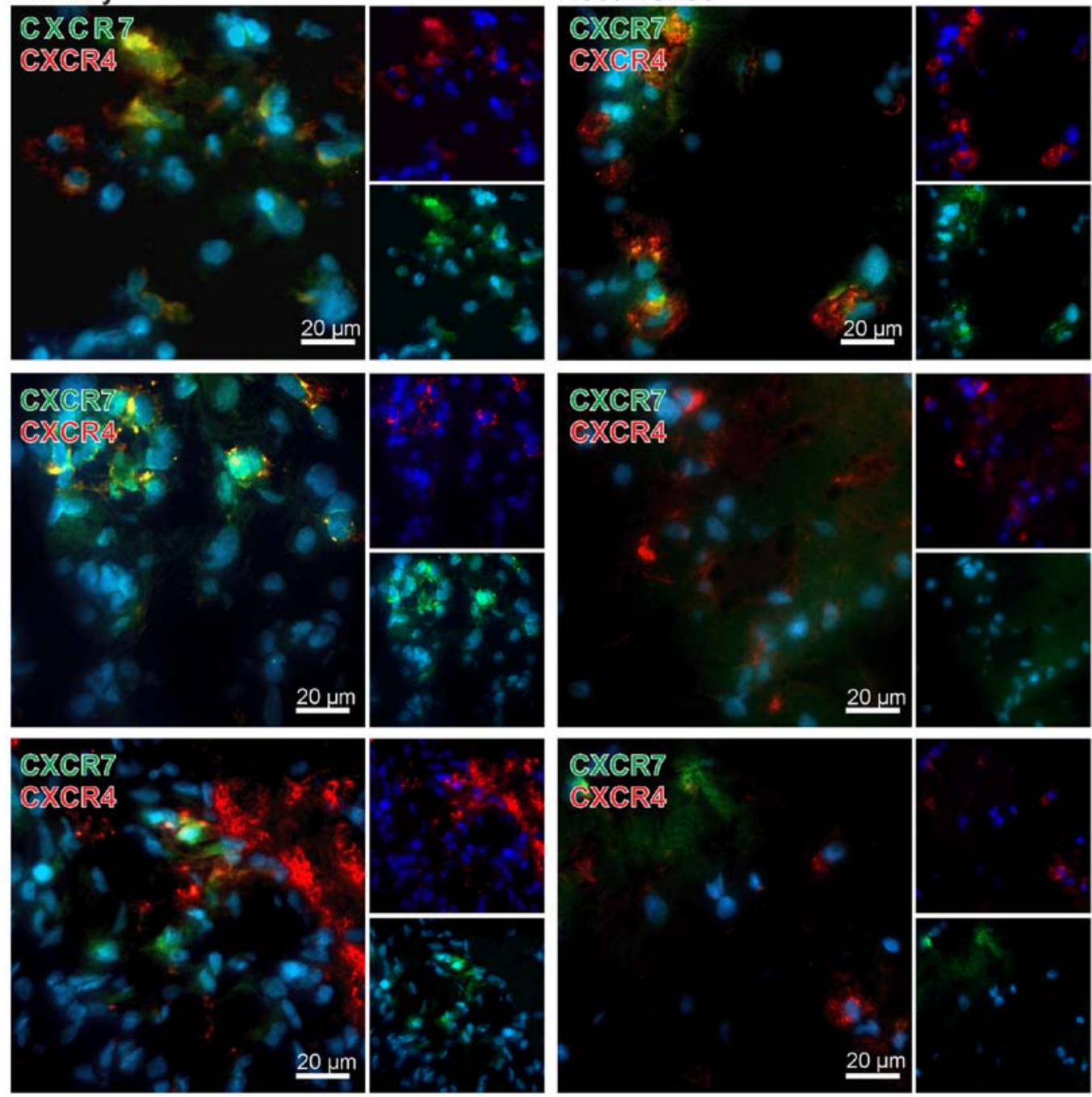

Figure 2. Expression of CXCR4 (red) and CXCR7 (green) in primary and recurrent glioblastoma as determined by immunofluorescence microscopy. Whereas in primary tumors numerous CXCR4 or CXCR7 positively stained cells were detectable, in matched recurrent samples only few CXCR7-positive, but considerable amounts of CXCR4-positive cells were found. For both primary and recurrent samples only single cells were CXCR4 and CXCR7 double-positive (yellow). The majority of tumor cells were solely positive for CXCR4 or CXCR7, respectively. Magnification, x400; bar, $20 \mu \mathrm{m}$.

factors, and NANOG which initially was used as readout for efficient reprogramming of iPSCs (21)] were performed and analyzed by fluorescence microscopy.

In general, CXCR4 yielded clear co-stainings with different stem cells markers in both primary and recurrent samples (exemplified in Fig. 3). However, this method does not allow for a valid quantification of both staining intensities and amounts of positively stained cells. Additionally, different antigens are not localized in the same cellular structures, thus signals do not merge in all cases, but can be found in the same regions. Nevertheless, especially in the co-staining CXCR4-SOX2 nearly all CXCR4-positive cells were also SOX-2-positive. For KLF-4, OCT-4 and MUSASHI-1 we also observed clear co-stainings with CXCR4 in both primary and recurrent GBM, with OCT-4-CXCR4 double-positive cells being remarkably localized in cluster-like structures. However, also CXCR4, KLF-4, OCT-4 and MUSASHI-1 single-positive cells existed within the sections. For NANOG, a co-staining with CXCR4 was also observed but not as prominent as detected for the other stem cell markers (exemplified in Fig. 3).
In comparison to CXCR4 the results for CXCR7 were clearly different. With exception of the combination CXCR7-KLF-4 only very few CXCR7-stem cell marker double-positive cells were found in the GBM specimens (exemplified in Fig. 4). In detail, when looking at OCT-4 and SOX-2 in CXCR7-positive regions, some OCT-4 or SOX-2-positive cells (and here more obvious in recurrences) were found, respectively, but it seems that in the majority of the cases CXCR7 was expressed in different, OCT-4 or SOX-2 negative cells. In line with these results, a clear co-staining of CXCR7 with NANOG or MUSASHI-1 was not detectable although positive cells were found in CXCR7-positive cell regions. In contrast, KLF-4 and CXCR7 were found co-expressed in the same cells and, although this method does not allow a valid quantification, this was more prominent in recurrent samples (exemplified in Fig. 4). Nevertheless, also KLF-4 and CXCR7 single-positive cells were found in the tumor sections, respectively.

Summarized, CXCR4 was clearly expressed in stem cell marker positive cells in both primary and recurrent GBM samples, whereas CXCR7, with the exception of a clear 


\section{Primary}
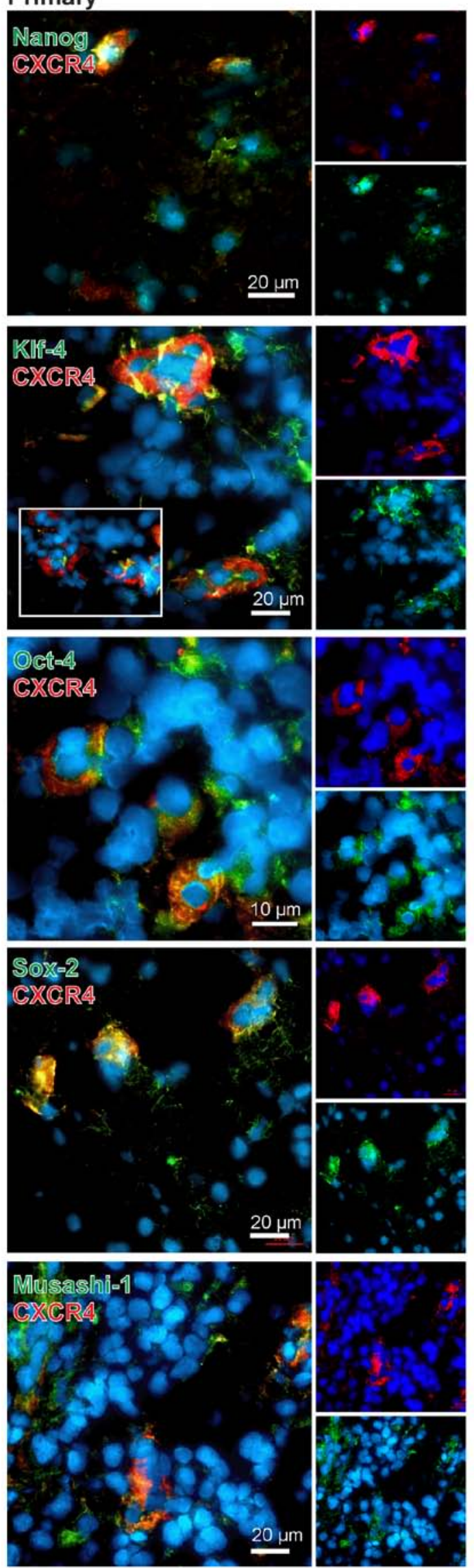

\section{Recurrence}
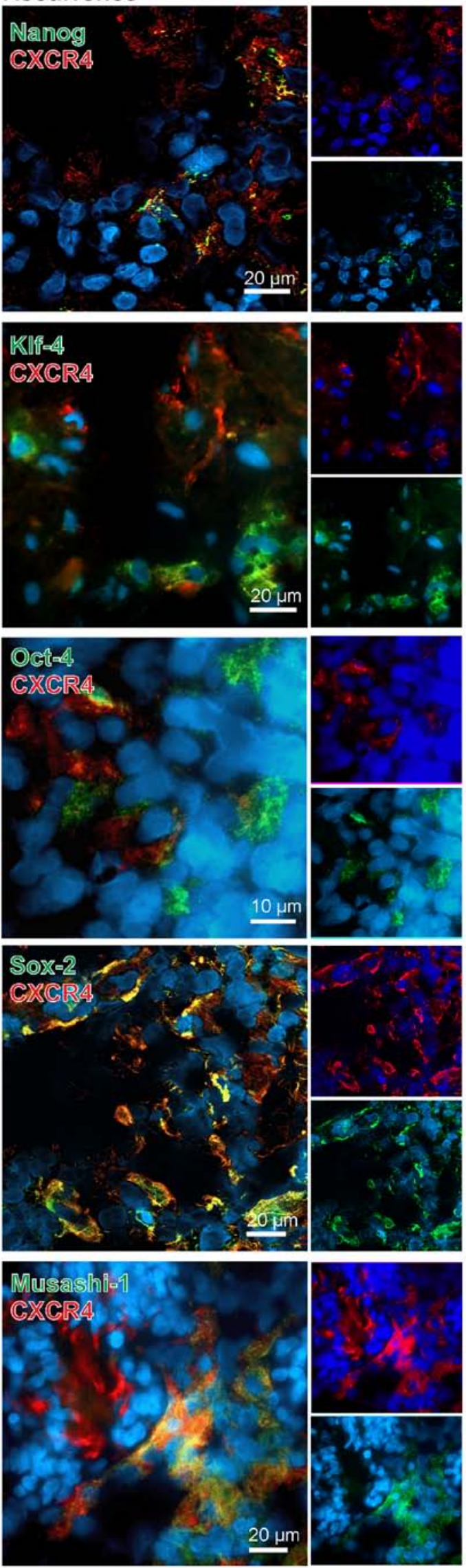

Figure 3. Representative co-staining of CXCR4 (red) with different stem cell markers (green) in primary and recurrent glioblastoma as determined by immunofluorescence microscopy. CXCR4 showed a clear co-staining with different stem cells markers in both primary and recurrent samples with most impressive results obtained for the combination of CXCR4-SOX2. Concerning the aspect that different markers are not all localized within the same structures in the cells, signals did not merge (yellow) in all cases. Nevertheless, also combinations of single-positive and double-negative cells were detectable. Magnification, $\mathrm{x} 400$; bar, $10-20 \mu \mathrm{m}$. 
Primary
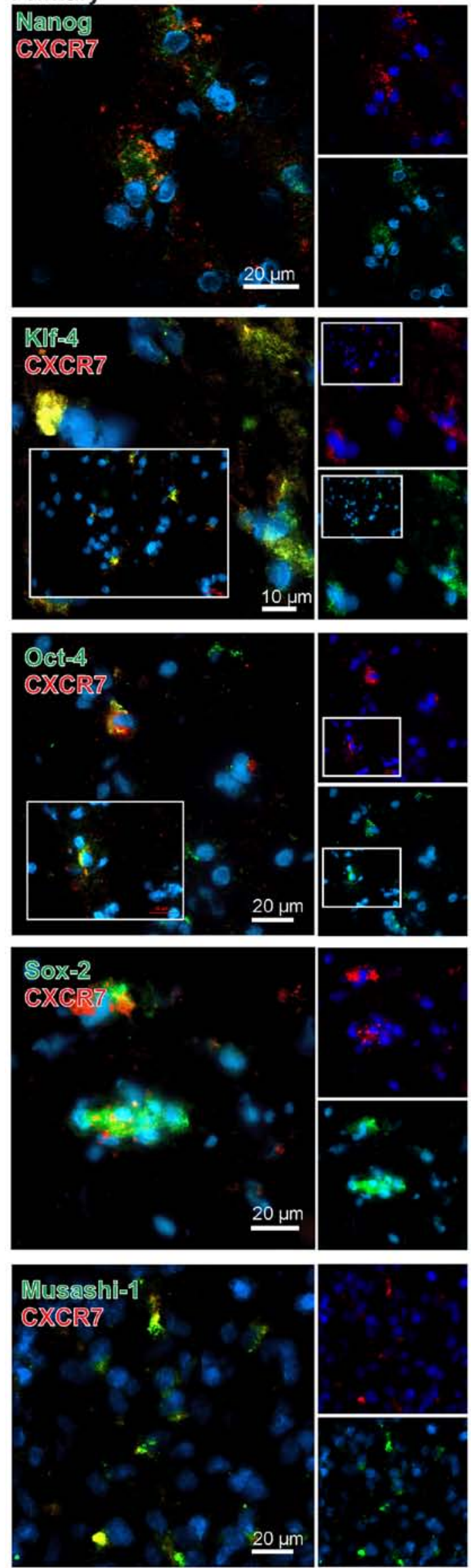

Recurrence
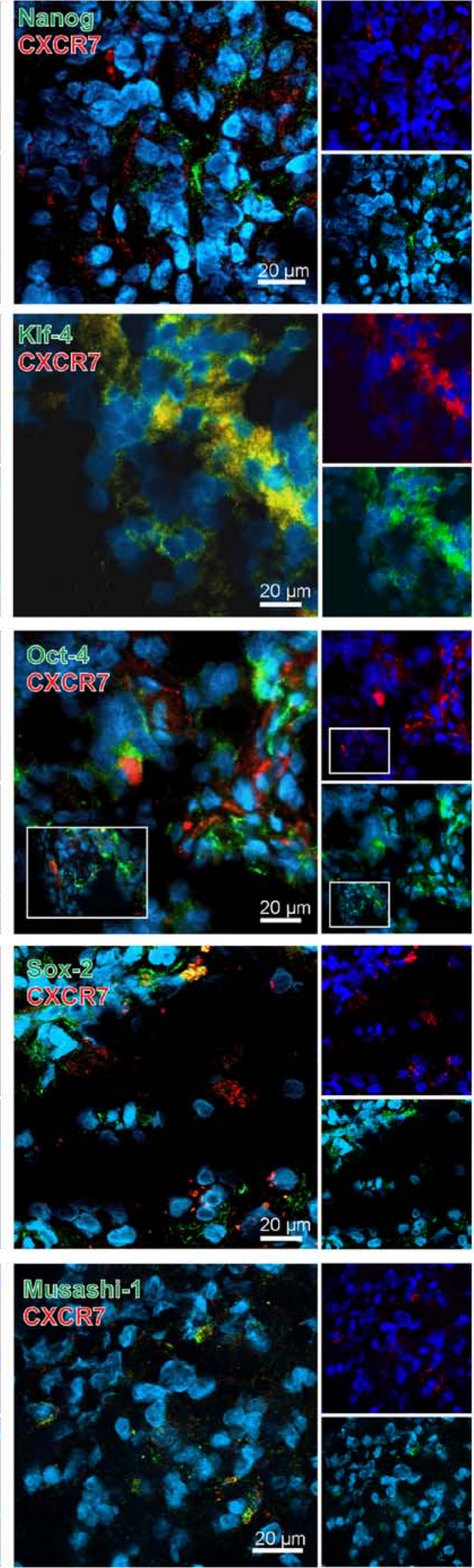

Figure 4. Representative co-staining of CXCR7 (red) with different stem cell markers (green) in primary and recurrent glioblastoma as determined by immunofluorescence microscopy. With exception of the combination CXCR7-KLF-4, only very few CXCR7-stem cell marker double-positive cells (yellow) were found in primary and recurrent GBM sections, respectively. The majority of the cells were only positive for CXCR7, but also KLF-4 and CXCR7 singlepositive cells were found in the tumor sections, respectively. Magnification, $\mathrm{x} 400$; bar, $20 \mu \mathrm{m}$. 
co-staining with KLF-4-positive cells especially in recurrences, was predominantly found in stem cell marker negative cells in the investigated samples.

\section{Discussion}

The chemokine CXCL12/SDF-1 is involved in glioma progression $(10,13,15,17)$. In GBM, CXCL12 and its receptor, CXCR4, are localized in regions of necrosis and angiogenesis (22), and are supposed to mediate proliferation of GBM progenitor cells (14). In contrast, the CXCL12 receptor CXCR7, which was initially regarded as a decoy receptor scavenging CXCL12 to prevent CXCR4 signaling and effects (23), mediates apoptosis resistance in human and rat glioma cells $(15,17)$. Previous results focusing on the expression of both receptors in human GBM indeed yielded an expression of CXCR4 by a subpopulation of GBM cells with stem cell properties (14), whereas the bulk of more differentiated GBM cells express CXCR7 $(15,24)$. Conversely, other studies reported the existence of CXCR4-CXCR7 double-positive cells and showed that this subpopulation might regulate the stem cell phenotype (25), and was able to initiate intracranial tumors in vivo (26). Nevertheless, also CXCR4 and CXCR7 single populations exist and a high level of heterogeneity in both the surface expression and functions of CXCR4 and CXCR7 in primary human GBM cells of the proliferative subclass was determined (26). In addition, GBM primary cell cultures show a heterogeneous cell surface expression of CXCR4 and CXCR7 despite similar levels of corresponding mRNAs (25), and the expression of CXCR4 and CXCR7 is significantly correlated (19).

Since comparative investigations regarding the expression of CXCR4 and CXCR7 in matched samples of primary and recurrent GBM are still lacking, we analysed this point in 14 different human primary-recurrent GBM pairs. We were able to show that irrespective of used materials (primary/recurrent GBM) both CXCR4 and CXCR7 were expressed at considerable amounts on the mRNA level with CXCR7 clearly to higher extent. Whereas CXCR4 expression was nearly equal in primary and recurrent samples, CXCR7 was found in lower amounts in recurrent GBM samples $(\sim 40 \%)$ in comparison to primary ones. Nevertheless when analyzing each individual pair in detail, two primaryrecurrent pairs were observed in our GBM collective with higher CXCR7 mRNA level in the recurrent samples. Although immunofluorescence staining of cryo-sections cannot clearly deliver quantitative data, the CXCR7 expression appeared lower in recurrent samples on the protein level, which is in accordance with the results obtained by qRT-PCR.

Interestingly, Razmkhah et al (27) reported that CXCR7 was undetectable in secondary brain tumors (sarcoma and breast cancer), whereas CXCR4 was expressed. Conversely to our observations, in that glioma cohort, CXCR4 expression was $~ 110$-fold higher than its counterpart CXCR7. However, only 7 samples of glioma (4 high-grade gliomas and 3 lowgrade gliomas without any histopathological specification) were included.

Further, when investigating CXCR4 and CXCR7 expression in primary and recurrent GBM on the protein level by doubleimmunofluorescence technique, we found that the chemokine receptors were mainly detectable on different cell types, only very few CXCR4-CXCR7 double-positive cells existed in the samples. In accordance with this, heterogeneity of CXCR4 and CXCR7 expression was reported before for primary GBM cells pointing to the existence of different subpopulations measurable by variable percentages of $\mathrm{CXCR} 4^{+} \mathrm{CXCR} 7^{-}$, CXCR4 4 CXCR $7^{+}$and $\mathrm{CXCR} 4^{+} \mathrm{CXCR} 7^{+}$cells in glioma samples (26). In addition, since for GBM-positive correlations not only for the expression of CXCR7 and CXCR4 but also for the expression of CXCR7 and HIF1 $\alpha$ and CXCR7 and IDH1 were reported, these results also indicate the existence of different chemokine receptor expressing subclones (19). By co-staining of CXCR4 and CXCR7 with stem cell markers we could show in our primary-recurrent GBM cohort that CXCR4 was mostly found on stem-cell marker positive GBM cells, whereas CXCR7 was usually expressed on stem-cell marker negative cells, with exception of the combination CXCR7-KLF-4. An embryonic stem cell gene signature is well known to correlate with a more undifferentiated phenotype in various cancers (28), and a large scale tissue microarray analysis including 80 low-grade and 98 high-grade gliomas showed an upregulated protein level of NANOG, KLF-4, OCT-4 and SOX-2 in highgrade gliomas (29). Several other studies also point to the relevance of neural and embryonic stem cell markers in GBM progression (30-36). In the present study we showed that both neural and embryonic stem cells markers are preferentially co-expressed with CXCR4 in matched samples of primary and recurrent GBM pairs, whereas CXCR7 was mostly found on stem cell marker negative cells. In line with these results, CXCR4 expression in neuroblastoma is associated with highly aggressive undifferentiated tumors, while CXCR7 expression was detected in more differentiated and mature neuroblastic tumors (37). In addition, CXCR4 is essential for the self-renewal of GBM stem-like cells since disruption of the CXCL12/CXCR4 pathway resulted in reduced expression of stem-like markers like Nestin and MUSASHI-1 or genes which are involved in regulating stem cell properties such as OCT-4 and NANOG (38). The miR-137 inhibited glioma stem-like cell self-renewal and promoted their differentiation by targeting RTVP-1, which downregulated CXCR4 (39). The inhibition of CXCR4 in glioma-initiating cells disrupted the SHH-GLI-NANOG network, which is involved in self-renewal and expression of the embryonic stem cell-like signature (40).

Interestingly, Chen et al (41) reported that CXCR7 can mediate neural progenitor cell migration to CXCL12 independently of CXCR4. In line with this, our primary-recurrent GBM cohort showed a distinct subpopulation of CXCR7-KLF-4 double-positive cells, which was more prominent in the recurrences. Since it has been shown that the miR-152 targets KLF-4 in GBM cells and thereby influences cell proliferation, invasion, apoptosis and cell migration processes (42), the CXCR7-KLF-4 double-positive GBM cells in our cohort could be a subpopulation of highly migratory tumor cells. However, the paracrine PGI signaling initiated by mesenchymal glioma cells also induces self-renewal and tumorigenic potential of glioma stem cells through induction of KLF-4 (43). The functional role of CXCR7 in KLF-4-positive glioma-stem cells remains still highly speculative.

Summarized, we were able to show that in a cohort of matched primary-recurrent GBM samples the CXCL12 receptors CXCR4 and CXCR7 are expressed both on the mRNA and protein levels in large amounts, with CXCR7 mRNA being 
statistically significantly downregulated in recurrent samples. A co-expression of both receptors was rare. In accordance with this, CXCR4 was co-expressed with all investigated neural and embryonic stem cell markers in both primary and recurrent tissues, whereas CXCR7 was mostly found on stem cell marker-negative cells but was co-expressed with KLF-4 on a distinct GBM cell subpopulation. These results point to an individual role of CXCR4 and CXCR7 in the contribution of stem cell marker-positive GBM cells in glioma progression and underline the potential of chemokine receptors as targets for new therapeutic approaches in GBM intervention.

\section{Acknowledgements}

We thank Brigitte Rehmke, Fereshteh Ebrahim and Jörg Krause for expert technical assistance. This study was supported by the University of Kiel and by the popgen 2.0 network [(P2N; supported by a grant from the German Ministry for Education and Research (01EY1103)].

\section{References}

1. Ohgaki H and Kleihues P: Epidemiology and etiology of gliomas Acta Neuropathol 109: 93-108, 2005.

2. Stupp R, Mason WP, van den Bent MJ, Weller M, Fisher B, Taphoorn MJ, Belanger K, Brandes AA, Marosi C, Bogdahn U, et al; European Organisation for Research and Treatment of Cancer Brain Tumor and Radiotherapy Groups; National Cancer Institute of Canada Clinical Trials Group: Radiotherapy plus concomitant and adjuvant temozolomide for glioblastoma. N Engl J Med 352: 987-996, 2005.

3. Strieter RM, Polverini PJ, Kunkel SL, Arenberg DA, Burdick MD, Kasper J, Dzuiba J, Van Damme J, Walz A, Marriott D, et al: The functional role of the ELR motif in CXC chemokine-mediated angiogenesis. J Biol Chem 270: 27348-27357, 1995.

4. Broxmeyer HE and Kim CH: Regulation of hematopoiesis in a sea of chemokine family members with a plethora of redundant activities. Exp Hematol 27: 1113-1123, 1999.

5. Dambly-Chaudière $\mathrm{C}$, Cubedo $\mathrm{N}$ and Ghysen A: Control of cell migration in the development of the posterior lateral line: Antagonistic interactions between the chemokine receptors CXCR4 and CXCR7/RDC1. BMC Dev Biol 7: 23, 2007.

6. Hattermann K, Ludwig A, Gieselmann V, Held-Feindt J and Mentlein R: The chemokine CXCL16 induces migration and invasion of glial precursor cells via its receptor CXCR6. Mol Cell Neurosci 39: 133-141, 2008.

7. Vandercappellen J, Van Damme J and Struyf S: The role of CXC chemokines and their receptors in cancer. Cancer Lett 267: 226-244, 2008.

8. O'Hayre M, Salanga CL, Handel TM and Allen SJ: Chemokines and cancer: Migration, intracellular signalling and intercellular communication in the microenvironment. Biochem J 409: 635-649, 2008

9. Müller A, Homey B, Soto H, Ge N, Catron D, Buchanan ME, McClanahan T, Murphy E, Yuan W, Wagner SN, et al: Involvement of chemokine receptors in breast cancer metastasis. Nature 410: 50-56, 2001.

10. Hattermann K and Mentlein R: An infernal trio: The chemokine CXCL12 and its receptors CXCR4 and CXCR7 in tumor biology. Ann Anat 195: 103-110, 2013.

11. Zlotnik A, Burkhardt AM and Homey B: Homeostatic chemokine receptors and organ-specific metastasis. Nat Rev Immunol 11: 597-606, 2011.

12. Bian XW, Yang SX, Chen JH, Ping YF, Zhou XD, Wang QL, Jiang XF, Gong W, Xiao HL, Du LL, et al: Preferential expression of chemokine receptor CXCR4 by highly malignant human gliomas and its association with poor patient survival. Neurosurgery 61: 570-579, 2007.

13. Würth R, Bajetto A, Harrison JK, Barbieri F and Florio T: CXCL12 modulation of CXCR4 and CXCR7 activity in human glioblastoma stem-like cells and regulation of the tumor microenvironment. Front Cell Neurosci 8: 144, eCollection, 2014.
14. Ehtesham M, Mapara KY, Stevenson CB and Thompson RC: CXCR4 mediates the proliferation of glioblastoma progenitor cells. Cancer Lett 274: 305-312, 2009.

15. Hattermann K, Held-Feindt J, Lucius R, Müerköster SS, Penfold ME, Schall TJ and Mentlein R: The chemokine receptor CXCR7 is highly expressed in human glioma cells and mediates antiapoptotic effects. Cancer Res 70: 3299-3308, 2010.

16. Auffinger B, Spencer D, Pytel P, Ahmed AU and Lesniak MS: The role of glioma stem cells in chemotherapy resistance and glioblastoma multiforme recurrence. Expert Rev Neurother 15: 741-752, 2015.

17. Hattermann K, Mentlein R and Held-Feindt J: CXCL12 mediates apoptosis resistance in rat C6 glioma cells. Oncol Rep 27: 1348-1352, 2012.

18. Liu Y, Carson-Walter EB, Cooper A, Winans BN, Johnson MD and Walter KA: Vascular gene expression patterns are conserved in primary and metastatic brain tumors. J Neurooncol 99: 13-24, 2010.

19. Bianco AM, Uno M, Oba-Shinjo SM, Clara CA, de Almeida Galatro TF, Rosemberg S, Teixeira MJ and Nagahashi Marie SK: CXCR7 and CXCR4 expression in infiltrative astrocytomas and their interactions with HIF1 $\alpha$ expression and IDH1 mutation. Pathol Oncol Res 21: 229-240, 2015.

20. Kubelt C, Hattermann K, Sebens S, Mehdorn HM and HeldFeindt J: Epithelial-to-mesenchymal transition in paired human primary and recurrent glioblastomas. Int J Oncol 46: 2515-2525, 2015.

21. Takahashi K and Yamanaka S: Induction of pluripotent stem cells from mouse embryonic and adult fibroblast cultures by defined factors. Cell 126: 663-676, 2006.

22. Rempel SA, Dudas S, Ge S and Gutiérrez JA: Identification and localization of the cytokine SDF1 and its receptor, CXC chemokine receptor 4 , to regions of necrosis and angiogenesis in human glioblastoma. Clin Cancer Res 6: 102-111, 2000.

23. Naumann U, Cameroni E, Pruenster M, Mahabaleshwar H, Raz E, Zerwes HG, Rot A and Thelen M: CXCR/functions as a scavenger for CXCR12 and CXCL11. Plos One 5: e9175, 2010.

24. Gatti M, Pattarozzi A, Bajetto A, Würth R, Daga A, Fiaschi P, Zona G, Florio $T$ and Barbieri F: Inhibition of CXCL12/CXCR4 autocrine/paracrine loop reduces viability of human glioblastoma stem-like cells affecting self-renewal activity. Toxicology 314: 209-220, 2013.

25. Walters MJ, Ebsworth K, Berahovich RD, Penfold ME, Liu SC, Al Omran R, Kioi M, Chernikova SB, Tseng D, MulkearnsHubert EE, et al: Inhibition of CXCR7 extends survival following irradiation of brain tumours in mice and rats. Br J Cancer 110: 1179-1188, 2014.

26. Liu C, Pham K, Luo D, Reynolds BA, Hothi P, Foltz G and Harrison JK: Expression and functional heterogeneity of chemokine receptors CXCR4 and CXCR7 in primary patientderived glioblastoma cells. PLoS One 8: e59750, 2013.

27. Razmkhah M, Arabpour F, Taghipour M, Mehrafshan A, Chenari $\mathrm{N}$ and Ghaderi A: Expression of chemokines and chemokine receptors in brain tumor tissue derived cells. Asian Pac J Cancer Prev 15: 7201-7205, 2014.

28. Ben-Porath I, Thomson MW, Carey VJ, Ge R, Bell GW, Regev A and Weinberg RA: An embryonic stem cell-like gene expression signature in poorly differentiated aggressive human tumors. Nat Genet 40: 499-507, 2008.

29. Elsir T, Edqvist P-H, Carlson J, Ribom D, Bergqvist M, Ekman S, Popova SN, Alafuzoff I, Ponten F, Nistér M, et al: A study of embryonic stem cell-related proteins in human astrocytomas: Identification of Nanog as a predictor of survival. Int J Cancer 134: 1123-1131, 2014.

30. Berezovsky AD, Poisson LM, Cherba D, Webb CP, Transou AD, Lemke NW, Hong X, Hasselbach LA, Irtenkauf SM, Mikkelsen T, et al: Sox 2 promotes malignancy in glioblastoma by regulating plasticity and astrocytic differentiation. Neoplasia 16: 193-206, 206.e19-206.e25, 2014.

31. Du Z, Jia D, Liu S, Wang F, Li G, Zhang Y, Cao X, Ling EA and Hao A: Oct4 is expressed in human gliomas and promotes colony formation in glioma cells. Glia 57: 724-733, 2009.

32. Galatro TF, Uno M, Oba-Shinjo SM, Almeida AN, Teixeira MJ, Rosemberg S and Marie SK: Differential expression of ID4 and its association with TP53 mutation, SOX2, SOX4 and OCT-4 expression levels. PLoS One 8: e61605, 2013.

33. Guo Y, Liu S, Wang P, Zhao S, Wang F, Bing L, Zhang Y, Ling EA, Gao J and Hao A: Expression profile of embryonic stem cell-associated genes Oct4, Sox 2 and Nanog in human gliomas. Histopathology 59: 763-775, 2011. 
34. Holmberg J, He X, Peredo I, Orrego A, Hesselager G, Ericsson C, Hovatta O, Oba-Shinjo SM, Marie SK, Nistér M, et al: Activation of neural and pluripotent stem cell signatures correlates with increased malignancy in human glioma. PLoS One 6: e18454, 2011.

35. Ikushima H, Todo T, Ino Y, Takahashi M, Saito N, Miyazawa K and Miyazono K: Glioma-initiating cells retain their tumorigenicity through integration of the Sox axis and Oct4 protein. J Biol Chem 286: 41434-41441, 2011.

36. Muto J, Imai T, Ogawa D, Nishimoto Y, Okada Y, Mabuchi Y, Kawase T, Iwanami A, Mischel PS, Saya H, et al: RNA-binding protein Musashil modulates glioma cell growth through the post-transcriptional regulation of Notch and PI3 kinase/Akt signaling pathways. PLoS One 7: e33431, 2012.

37. Mühlethaler-Mottet A, Liberman J, Ascenção K, Flahaut M, Balmas Bourloud K, Yan P, Jauquier N, Gross N and Joseph JM: The CXCR4/CXCR7/CXCL12 axis is involved in a secondary but complex control of neuroblastoma metastatic cell homing. PLoS One 10: e0125616, 2015.

38. Lee CC, Lai JH, Hueng DY, Ma HI, Chung Y, Sun YY, Tsai YJ, Wu WB and Chen CL: Disrupting the CXCL12/CXCR4 axis disturbs the characteristics of glioblastoma stem-like cells of rat RG2 glioblastoma. Cancer Cell Int 13: 85, 2013.

39. Bier A, Giladi N, Kronfeld N, Lee HK, Cazacu S, Finniss S Xiang C, Poisson L, deCarvalho AC, Slavin S, et al: MicroRNA-137 is downregulated in glioblastoma and inhibits the stemness of glioma stem cells by targeting RTVP-1. Oncotarget 4: 665-676, 2013.
40. Fareh M, Turchi L, Virolle V, Debruyne D, Almairac F, de-la- Forest Divonne S, Paquis P, Preynat-Seauve O, Krause K-H, Chneiweiss $\mathrm{H}$, et al: The miR 302-367 cluster drastically affects self-renewal and infiltration properties of glioma-initiating cells through CXCR4 repression and consequent disruption of the SHH-GLI-NANOG network. Cell Death Differ 19: 232-244, 2012.

41. Chen Q, Zhang M, Li Y, Xu D, Wang Y, Song A, Zhu B, Huang Y and Zheng JC: CXCR7 mediates neural progenitor cells migration to CXCL12 independent of CXCR4. Stem Cells 33: 2574-2585, 2015.

42. Ma J, Yao Y, Wang P, Liu Y, Zhao L, Li Z, Li Z and Xue Y: MiR-152 functions as a tumor suppressor in glioblastoma stem cells by targeting Krüppel-like factor 4. Cancer Lett 355: 85-95, 2014.

43. Zhu XY, Wang L, Luan SH, Zhang HS, Huang WT and Wang NH: The PGI-KLF4 pathway regulates self-renewal of glioma stem cells residing in the mesenchymal niches in human gliomas. Neoplasma 61: 401-410, 2014. 